\title{
The Impact of Body Mass Index on Intraoperative Blood Loss, Blood Transfusion and Fluid Management in Patients Undergoing Liver Transplantation: A Retrospective Analysis from a Tertiary Referral Center
}

\author{
(1) Tumay Uludag Yanaral, (1) Pelin Karaaslan \\ Istanbul Medipol University Faculty of Medicine, Department of Anesthesiology and Reanimation, Istanbul, Turkey
}

\section{Abstract}

\begin{abstract}
Aim: Some parameters affecting intraoperative bleeding have been investigated, but there is not enough data on body mass index (BMI) and bleeding in liver transplantation (LT). We aimed to evaluate the relationship between the pretransplant BMI of recipients and blood loss, blood product transfusion, and fluid replacement during LT.
\end{abstract}

Methods: In this retrospective cross-sectional study, patients aged $\geq 18$ years who underwent LT between April 2014 and June 2020 were analyzed. Patients $<18$ years of age, with incomplete data, and those using cell salvage were excluded from the study. The BMI of the patients was grouped according to the definition of the World Health Organization (BMI <18.5 in Group 1, BMI 18.5-24.9 in Group 2, BMI 25-29.9 in Group 3, and BMI >30 in Group 4). Groups were compared according to operative hemodynamics, blood loss, blood transfusion, and fluid management-related parameters.

Results: Two hundred and sixteen patients were included in the study. The mean blood loss was higher in obese patients than in others, but it was not statistically significant. The mean red blood cell, fresh frozen plasma, platelet, and cryoprecipitate transfusions were similar for the groups. The mean IV crystalloid fluid amount gradually shortened from underweight to obese patients but there was no significant difference. Preoperative mean international normalized ratio and prothrombin time were significantly higher in underweight patients than others $(p=0.025)$.

Conclusion: LT can be performed safely in patients with different BMI with similar blood loss and transfusion rates.

Keywords: Anesthesia, blood transfusion, body mass index, hemorrhage, liver transplantation, obesity

\section{Introduction}

Liver transplantation (LT) is a life-saving procedure for patients with end-stage liver disease (ESLD). It is a complex and technically difficult surgery with multiple vascular transactions and anastomoses that increase the risk of blood loss. Although IV fluids are used in the first step of blood loss management, sometimes massive blood transfusion may be required. To make proper patient preparation before surgery, it is very important to be able to identify patients with a high probability of bleeding during LT and anticipate their transfusion needs. In this way, a prediction can be formed for both the preparation of blood products and the timing of the transplantation (1).
The increase in the overweight population is an important health problem worldwide. Obesity is known to be a risk factor for the development of diabetes mellitus, hypertension, and coronary artery disease. It is also thought to increase the perioperative morbidity and mortality resulting from surgery (2). The effects of body mass index (BMI) on clinical outcomes, complications, and perioperative parameters in surgery have been investigated before (3). It has also been reported that obesity is associated with higher infectious complications and cancer events leading to death in liver transplant recipients (4). 
Although some parameters affecting intraoperative bleeding have been investigated, there is not enough data about BMI and bleeding in LT. Therefore, in this study, we evaluated the relationship between the pretransplant BMI of recipients and blood loss, blood product transfusion, and fluid replacement during LT.

\section{Methods}

\section{Study design}

After obtaining approval from This study was approved by the Ethics Committee of the Istanbul Medipol University (approval number: 2021/126) the medical records of patients aged 18 years and over who underwent LT in our hospital between April 2014 and June 2020 were retrospectively analyzed. Data were collected using patients' charts and hospital medical records. Demographic data of the patients, parameters related to ESLD, anesthesia and surgery-related data, and laboratory test results were recorded. The exclusion criteria were being under the age of 18 , incomplete data, and cell salvage (autologous blood transfusion system) usage during surgery. Verbal consent was obtained by informing the participating patients or their legal guardians.

BMI was calculated by dividing weight $(\mathrm{kg})$ by square of height $(\mathrm{m})$ and the World Health Organization (WHO) definition was used in its classification. Patients were divided into 4 groups according to the body mass index, as the BMI <18.5 (underweight) in Group 1, BMI 18.5-24.9 (normal weight) in Group 2, BMI 25-29.9 (overweight) in Group 3, and the BMI >30 (obese) in Group 4. Groups were compared according to preoperative demographic characteristics, operative hemodynamics, blood loss, blood transfusion, and fluid management-related parameters.

\section{Anesthesia management}

General anesthesia was employed for all LT surgeries. After preoxygenation, general anesthesia was induced with IV propofol, fentanyl, and vecuronium. Sevoflurane (2-3\%) was used as an inhalation agent to maintain anesthesia. A central venous catheter was placed for fluid infusion, central venous pressure (CVP), and mixed venous oxygen saturation monitoring. A radial arterial line was used for blood pressure measurement. Intraoperative monitoring includes serial arterial blood gases, temperature, thromboelastogram (TEG), pulse index contour continuous cardiac output, and standard laboratory tests. We applied the restrictive fluid management technique before the anhepatic phase to reduce blood loss.

The transfusion strategy was recommended according to the guideline by the American Association of Blood Banks for transfusion. If the hemoglobin was below $7 \mathrm{~g} / \mathrm{dL}$, allogeneic red blood cell (RBC) transfusion was performed. For hemoglobin levels between 8 and $10 \mathrm{mg} / \mathrm{L}$, the decision to transfuse was made based on symptoms and signs of anemia. If bleeding continued, allogeneic fresh frozen plasma (FFP), platelet, and cryoprecipitate transfusion were used according to TEG results. All patients were transferred to the intensive care unit (ICU) for close follow-up after surgery.

\section{Statistical Analysis}

The normality distribution of the variables was checked by the Shapiro-Wilk test and Q-Q plots. One-Way ANOVA test was used for comparison of variables showing normal distribution between groups, and the Kruskal-Wallis test was used for data that did not show normal distribution. Quantitative data are showed as mean \pm standard deviation. Categorical variables were grouped and compared using the $\chi^{2}$ test or Fisher's Exact test. The data were analyzed at a $95 \%$ confidence level and a p-value of less than 0.05 was accepted as statistically significant.

\section{Results}

Two hundred and sixteen patients [Group 1=6 (2.8\%), Group 2=56 (25.9\%), Group 3=82 (38\%) and Group $4=72(33.3 \%)$ patients] were included in the study (Figure 1). In the study cohort, the highest number of patients, with a rate of $38 \%$, was in the overweight group. The mean ages were $47.5 \pm 12.8,47.9 \pm 14.3,54.4 \pm 10.0$, and $56.1 \pm 11.2$ years for Group 1, Group 2, Group 3, and Group 4, respectively $(p=0.001)$. Gender, diabetes mellitus, hypertension, coronary artery disease and model for endstage liver disease (MELD) score were comparable between the groups $(p=0.078, p=0.347, p=0.863, p=0.486$ and $p=0.119$, respectively) (Table 1 ). The most common cause of ESLD was the Hepatitis B virus (47 patients) and the second most common cause was cryptogenic cirrhosis (45 patients).

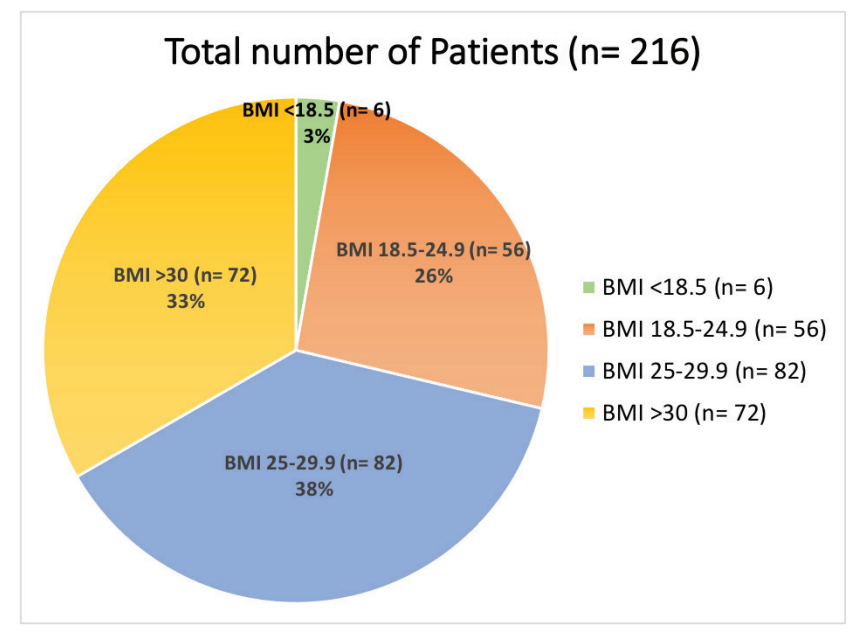

Figure 1. Number of patients according to groups BMI: Body mass index $\left(\mathrm{kg} / \mathrm{m}^{2}\right)$ 
Table 1. Demographic and clinical characteristics of the patients who underwent liver transplantation

\begin{tabular}{|l|l|l|l|l|l|}
\hline & BMI <18.5 (n=6) & BMI 18.5-24.9 (n=56) & BMI 25-29.9 (n=82) & BMI >30 (n=72) & p \\
\hline Age, years & $47.5 \pm 12.8$ & $47.9 \pm 14.3$ & $54.4 \pm 10.0$ & $56.1 \pm 11.2$ & $0.001^{*}$ \\
\hline Gender (Male/Female) & $2 / 4$ & $40 / 16$ & $62 / 20$ & $45 / 27$ & 0.078 \\
\hline Diabetes mellitus & $2(33.3 \%)$ & $11(19.6 \%)$ & $26(31.8 \%)$ & $25(34.7 \%)$ & 0.347 \\
\hline Hypertension & $0(0 \%)$ & $6(10.7 \%)$ & $6(7.3 \%)$ & $7(9.7 \%)$ & 0.863 \\
\hline Coronary artery disease & $0(0 \%)$ & $11(19.7 \%)$ & $13(15.9 \%)$ & $8(11.1 \%)$ & 0.486 \\
\hline MELD score & $22.5 \pm 8.1$ & $16.4 \pm 6.1$ & $16.8 \pm 6.4$ & $15.4 \pm 5.6$ & 0.119 \\
\hline BMI: Body mass index $\left(\mathrm{kg} / \mathrm{m}^{2}\right)$, & MELD: Model for end-stage liver disease, ${ }^{*}$ Kruskal-Wallis test & & \\
\hline
\end{tabular}

Table 2. Comparison of anesthesia and surgery related parameters according to the body mass index of the patients

\begin{tabular}{|c|c|c|c|c|c|}
\hline & BMI $<18.5(n=6)$ & BMI 18.5-24.9 $(n=56)$ & BMI 25-29.9 $(n=82)$ & $\mathrm{BMI}>30(n=72)$ & $p$ \\
\hline Donor type (Living/deceased) & $6 / 0$ & $47 / 9$ & $74 / 8$ & $56 / 16$ & 0.152 \\
\hline Cold ischemia time, minutes & $38.5 \pm 20.9$ & $114.0 \pm 151.8$ & $77.7 \pm 92.5$ & $105.5 \pm 115.7$ & 0.320 \\
\hline Warm ischemia time, minutes & $33.0 \pm 21.9$ & $39.4 \pm 22.8$ & $42.6 \pm 22.6$ & $44.6 \pm 25.4$ & 0.515 \\
\hline Urine output, $\mathrm{mL}$ & $3108.3 \pm 1437.5$ & $2454.1 \pm 1568.7$ & $2392.9 \pm 1399.9$ & $2264.3 \pm 1425.0$ & 0.460 \\
\hline \multicolumn{6}{|l|}{$\mathrm{CVP}\left(\mathrm{cm} \mathrm{H}_{2} \mathrm{O}\right)$} \\
\hline At the induction & $8.7 \pm 4.5$ & $11.3 \pm 3.6$ & $10.9 \pm 4.3$ & $12.1 \pm 3.4$ & 0.320 \\
\hline At the extubation & $5.0 \pm 1.7$ & $9.7 \pm 3.7$ & $9.0 \pm 2.7$ & $9.2 \pm 2.8$ & 0.104 \\
\hline Blood loss, $\mathrm{mL}$ & $2449.7 \pm 1470.5$ & $2862.4 \pm 3329.6$ & $2412.9 \pm 2186.3$ & $3094.4 \pm 3454.6$ & 0.636 \\
\hline \multicolumn{6}{|l|}{ Intraoperative transfusions, unit } \\
\hline Red blood cell & $3.5 \pm 3.2$ & $3.9 \pm 4.0$ & $3.0 \pm 2.9$ & $3.7 \pm 3.4$ & 0.415 \\
\hline Fresh frozen plasma & $7.1 \pm 5.3$ & $7.7 \pm 9.0$ & $6.8 \pm 4.9$ & $8.6 \pm 6.9$ & 0.615 \\
\hline Platelet & $0.2 \pm 0.5$ & $0.9 \pm 1.5$ & $0.6 \pm 1.0$ & $1.0 \pm 1.6$ & 0.704 \\
\hline Cryoprecipitate & 0 & $1.0 \pm 2.3$ & $1.1 \pm 1.9$ & $0.9 \pm 1.6$ & 0.372 \\
\hline Crystalloid fluid amount, $\mathrm{mL}$ & $8000.0 \pm 4147.3$ & $6631.2 \pm 3022.4$ & $6227.3 \pm 2400.3$ & $6212.1 \pm 3490.3$ & 0.467 \\
\hline Colloid fluid amount, $\mathrm{mL}$ & $400.0 \pm 223.6$ & $448.6 \pm 253.5$ & $475.4 \pm 293.6$ & $560.4 \pm 354.8$ & 0.549 \\
\hline Anesthesia duration, hours & $8.0 \pm 1.7$ & $7.9 \pm 1.5$ & $7.9 \pm 1.7$ & $7.9 \pm 1.9$ & 0.939 \\
\hline Length of stay in ICU, days & $2.8 \pm 1.3$ & $2.8 \pm 1.9$ & $2.3 \pm 1.7$ & $3.5 \pm 4.3$ & 0.081 \\
\hline Length of stay in hospital, days & $19.3 \pm 8.9$ & $16.4 \pm 9.7$ & $17.7 \pm 12.1$ & $16.2 \pm 12.8$ & 0.669 \\
\hline
\end{tabular}

Donor type, cold ischemia time, warm ischemia time, urine output, CVP at induction and at extubation, anesthesia duration, length of stay in ICU and length of stay in hospital were similar between the groups $(p=0.152$, $p=0.320, p=0.515, p=0.460, p=0.320, p=0.104, p=0.939$, $p=0.081$ and $p=0.669$, respectively). The mean blood loss was higher in Group 4 (3094.4 mL) than in other groups, but it was not statistically significant between the groups $(p=0.636)$. The mean RBC, FFP, platelet and cryoprecipitate transfusions were also similar for the groups $(p=0.415$, $p=0.615, p=0.704$ and $p=0.372$, respectively). The mean IV crystalloid fluid amount gradually shortened from group 1 to group 4 but there was no significant difference between the groups (8000, 6631.2, 6227.3, and $6212.1 \mathrm{ml}$, respectively, $\mathrm{p}=0.467)$. Similarly, groups were comparable in terms of colloid fluid replacement $(p=0.549)$ (Table 2).
The comparison of laboratory parameters is shown in Table 3. The mean platelet count, hemoglobin, activated partial thromboplastin time and fibrinogen were all similar between the groups. All these parameters did not differ between the groups in the preoperative and postoperative periods. The mean preoperative international normalized ratio (INR) was 2, 1.5, 1.8, and 1.5 for Group 1, Group 2, Group 3, and Group 4, respectively $(p=0.033)$. Also, preoperative mean prothrombin time (PT) was significantly higher in Group 1 than in other groups $(p=0.025)$. Both postoperative INR and PT were comparable between the groups ( $p=0.896$ and $p=0.711$, respectively).

\section{Discussion}

Obesity is an important public health problem with an increasing worldwide prevalence. Globally, obesity has nearly tripled in the last 40 years. According to $\mathrm{WHO}$, $39 \%$ of adults were overweight and $13 \%$ were obese in 
Uludag Yanaral and Karaaslan. Impact of BMI on Liver Transplant Anesthesia

\begin{tabular}{|c|c|c|c|c|c|}
\hline & BMI $<18.5(n=6)$ & BMI 18.5-24.9 $(n=56)$ & BMI 25-29.9 $(n=82)$ & $\mathrm{BMI}>30(n=72)$ & $\mathbf{p}$ \\
\hline \multicolumn{6}{|c|}{ Platelet count $\left(1000 / \mathrm{mm}^{3}\right)$} \\
\hline Preoperative & $148.2 \pm 84.9$ & $123.4 \pm 103.0$ & $87.3 \pm 47.3$ & $90.6 \pm 54.9$ & 0.136 \\
\hline Postoperative & $10.8 .0 \pm 59.4$ & $74.8 \pm 52.8$ & $62.9 \pm 36.8$ & $58.4 \pm 28.3$ & 0.360 \\
\hline \multicolumn{6}{|c|}{ Hemoglobin $(g / d L)$} \\
\hline Preoperative & $9.9 \pm 1.4$ & $10.2 \pm 1.9$ & $10.6 \pm 2.2$ & $10.9 \pm 2.0$ & 0.375 \\
\hline Postoperative & $8.1 \pm 0.1$ & $8.4 \pm 1.4$ & $8.5 \pm 1.3$ & $8.9 \pm 1.7$ & 0.610 \\
\hline \multicolumn{6}{|l|}{ INR } \\
\hline Preoperative & $2.0 \pm 1.4$ & $1.5 \pm 0.4$ & $1.8 \pm 0.9$ & $1.5 \pm 0.4$ & $0.033^{*}$ \\
\hline Postoperative & $2.2 \pm 0.3$ & $2.2 \pm 0.9$ & $2.2 \pm 0.7$ & $2.0 \pm 0.5$ & 0.896 \\
\hline \multicolumn{6}{|c|}{ Prothrombin time (seconds) } \\
\hline Preoperative & $24.0 \pm 10.4$ & $17.5 \pm 3.9$ & $21.2 \pm 7.7$ & $18.8 \pm 5.3$ & $0.025^{*}$ \\
\hline Postoperative & $25.9 \pm 5.7$ & $23.0 \pm 8.5$ & $23.3 \pm 6.1$ & $22.6 \pm 5.4$ & 0.711 \\
\hline \multicolumn{6}{|c|}{ Activated partial thromboplastin time (seconds) } \\
\hline Preoperative & $28.3 \pm 3.3$ & $37.6 \pm 6.0$ & $37.9 \pm 2.6$ & $38.9 \pm 6.1$ & 0.086 \\
\hline Postoperative & $38.5 \pm 2.1$ & $54.1 \pm 40.4$ & $39.4 \pm 4.9$ & $48.2 \pm 22.5$ & 0.833 \\
\hline \multicolumn{6}{|c|}{ Fibrinogen (mg/dL) } \\
\hline Preoperative & $239.8 \pm 141.5$ & $273.4 \pm 97.0$ & $216.5 \pm 85.4$ & $244.8 \pm 111.4$ & 0.103 \\
\hline Postoperative & $118.0 \pm 32.5$ & $145.6 \pm 44.2$ & $111.9 \pm 34.5$ & $114.7 \pm 37.1$ & 0.116 \\
\hline
\end{tabular}

2016 (5). The rate of obese patients in LT recipients has also increased from $20 \%$ to $33 \%$ over the years (6). Nonalcoholic steatohepatitis, a consequence of obesity, is the fourth leading indication for LT and it is estimated to pass hepatitis $C$ as the main indication in the next years (7). In this study, the rates of overweight and obese patients were $38 \%$ and $33.3 \%$, respectively. Our rate of overweight patients was similar to population-based studies, and our obese patients' rate was higher than the overall prevalence as in other LT studies (6).

High BMI increases the risk of diseases such as cardiovascular and musculoskeletal diseases, and several cancers. It is also a predictor of decompensation of ESLD and is known to worsen portal hypertension (8). Regarding LT complications, it has been demonstrated that infectious and biliary complications are higher in obese patients (9). It can be thought that all these parameters may increase the duration of surgery and anesthesia. Obesity has been shown to increase the duration of surgery in some, but there are also studies reporting that it does not change the duration of surgery $(10,11)$. In a study conducted on 813 liver recipients, it was reported that the mean operation time in morbidly obese patients ( 8.2 hours) was significantly longer compared to those with normal weight and underweight patients (7.2 hours) (9). However, Conzen et al. (7) concluded that there was no difference between the BMI groups in terms of operation time. In our study, anesthesia durations were similar between the groups, and obesity was not a factor affecting the duration of anesthesia.
Transplantation is surgery with a high risk of bleeding due to portal hypertension and coagulopathy in ESLD patients. The amount of bleeding can vary from hospital to hospital depending on many variables such as the recipient's preoperative clinical status, the condition of the graft, and the surgical technique. In our study, the mean blood loss of the groups is 2412-3094 $\mathrm{ml}$ and is not affected by the BMI category. These results are contrary to some studies showing that the amount of bleeding increases as BMI increases, but they are consistent with some other studies $(7,9,12)$. We think that excessive fluid overload during LT is also one of the determining factors of bleeding. Therefore, we apply the restrictive fluid resuscitation strategy before the anhepatic phase in anesthesia management. Previously, preoperative blood products or agents have been used to correct an existing coagulopathy and fibrinolysis to reduce bleeding during LT (9). However, nowadays, we prefer to use these managements not preoperatively but during surgery, if needed.

Different results have been reported between obesity and blood transfusion in the literature. Although there are studies reporting that blood transfusion increases as $\mathrm{BMI}$ increases, there are also studies reporting that there is no difference $(6,13)$. In studies conducted in 2001 and 2005, they found RBC transfusion as 13-16 units for the obese group and 11-16 units for the non-obese patients. In these studies, there was no difference between the groups in terms of both RBC, platelet, and FFP transfusion 
$(12,14)$. Correspondingly, in our study, the number of intraoperative transfusions was similar between the groups. Over the years, blood transfusion rates have decreased due to the increase in anesthesia and surgical experience and improvement of medical technology. In all groups, we performed LT with an average of 3-3.9 units of RBC transfusion intraoperatively. One of the factors that increase the blood product transfusion is the prolonged donor ischemia time (15). Our study groups were similar in terms of both cold ischemia time and warm ischemia time. We think that this is also effective in the similarity of blood transfusion rates.

Various strategies have been adopted to limit the amount of blood transfusion, including the use of autotransfusion devices, avoiding excessive fluid administration, and maintaining relative hypovolemia. During liver resections, a low CVP through volume restriction has been shown to reduce surgical blood loss and transfusion rate (16). However, it is not clear what an optimal CVP value is to prevent bleeding during LT. Accordingly, we apply restrictive fluid therapy before hepatectomy in anesthesia management to prevent volume overload. Although in the current study there was no difference in the mean intraoperative fluid replacement amount between the groups, the crystalloid amount was higher in Group 1. We argue that this may be due to the higher MELD scores of the patients in Group 1. Feltracco et al. (16) reported that individualized proper intraoperative fluids and prevention and treatment of coagulation abnormalities had a positive effect on the amount of bleeding and transfusion requirements. We found that, with the same approach as Feltracco et al. (16) similar fluid management can be performed in patients with different body mass indexes.

Significant changes are observed in the biochemical variables of the hemostatic system in patients with ESLD. These changes include thrombocytopenia, platelet functional defects, low coagulation factors and inhibitor levels, and low fibrinolytic protein levels. Similarly, the preoperative platelet count, INR and PT values of the patients in our study were abnormal. Preoperative INR and PT values of the patients in Group 1 were significantly higher than the other groups. We allege that these differences might be due to the higher MELD scores and severity of ESLD of the patients in Group 1. In those with only hemostatic disorders without liver disease, these abnormalities usually indicate a bleeding tendency. But the situation is different in patients with ESLD and the approach to hemostasis is changing recently. It is thought that the abnormal coagulation tests seen in patients with liver disease do not definitely specify an increased bleeding tendency (17). In a study of 206 liver transplants, it was found that the most important factors regarding the number of RBC transfusions were preoperative platelet count and duration of surgery (18). In accordance with this study, we found that our groups were similar in terms of these two parameters and blood product transfusions were also similar. In addition to routine coagulation tests, TEG quickly assesses functional coagulation status. TEG has been shown to improve the detection of intraoperative coagulation profiles and reduce blood transfusion (19). For the stated benefits, we use TEG in blood transfusion management during LT.

Long ICU and hospital stay are indicators of increased morbidity and factors that increase costs. Recipient age, severity of ESLD, preoperative nutritional status, donor age and weight, and complications are risk factors predicting a longer hospital stay (20). Previous reports have shown inconsistent results regarding ICU and hospital stay after LT in obese patients $(7,21,22)$. This inconsistency can be explained by the differences in BMI categories in the groups, the attitudes of the institutes about the duration of discharge, and the number of patients in the studies. In our study, obesity is not a factor that affects on length of ICU and hospital stay. Our length of ICU stay was in concordance with a study by Conzen et al. (7) reporting 2-6 days, but our length of hospital stay was higher than their result which was 7-12 days.

\section{Study Limitations}

This study has its own limitations. First, the number of patients in the groups was not similar. Secondly, subgroup analysis was not performed (e.g., obese-nonobese, morbid obese-normal weight). Lastly, early postoperative blood transfusion data and graft-patient survival data were not included. These limitations should be considered in further studies.

\section{Conclusion}

Clear guidelines on the management of blood transfusion in obese patients during LT are lacking. In the present study, we found the same blood loss, blood transfusion, and fluid replacement between groups. We concluded that LT can be performed safely in patients with different BMI with similar blood loss and transfusion rates. Our results should be supported by prospective multicenter studies from different populations.

\section{Authorship Contributions}

Concept: T.U.Y., P.K., Design: T.U.Y., P.K., Data Collection or Processing: T.U.Y., Analysis or Interpretation: T.U.Y., P.K., Literature Search: T.U.Y., Writing: T.U.Y.

Conflict of Interest: No conflict of interest was declared by the authors.

Financial Disclosure: The authors declared that this study received no financial support. 


\section{References}

1. Pandey CK, Singh A, Kajal K, et al. Intraoperative blood loss in orthotopic liver transplantation: The predictive factors. World J Gastrointest Surg 2015;7:86-93.

2. Wang $M$, Chen $M$, Ao $H$, Chen S, Wang Z. The Effects of Different BMI on Blood Loss and Transfusions in Chinese Patients Undergoing Coronary Artery Bypass Grafting. Ann Thorac Cardiovasc Surg 2017;23:83-90.

3. Villavicencio A, Lee Nelson E, Rajpal S, Vivek N, Burneikiene S. The impact of BMI on operating room time, blood loss, and hospital stay in patients undergoing spinal fusion. Clin Neurol Neurosurg 2019;179:19-22.

4. Higuera-de-la-Tijera F, Córdova-Gallardo J, Buganza-Torio E, et al. Hepamet Fibrosis Score in Nonalcoholic Fatty Liver Disease Patients in Mexico: Lower than Expected Positive Predictive Value. Dig Dis Sci 2021;11.

5. World Health Organization. Obesity and overweight. Available from: https://www.who.int/news-room/fact-sheets/detail/ obesity-and-overweight (accessed March 2021).

6. Spengler EK, O'Leary JG, Te HS, et al. Liver Transplantation in the Obese Cirrhotic Patient. Transplantation 2017;101:228896.

7. Conzen KD, Vachharajani N, Collins KM, et al. Morbid obesity in liver transplant recipients adversely affects longterm graft and patient survival in a single-institution analysis. HPB (Oxford) 2015;17:251-7.

8. Nishikawa $\mathrm{H}$, Enomoto $\mathrm{H}$, Nishiguchi $\mathrm{S}$, lijima $\mathrm{H}$. Sarcopenic Obesity in Liver Cirrhosis: Possible Mechanism and Clinical Impact. Int J Mol Sci 2021;22:1917.

9. LaMattina JC, Foley DP, Fernandez LA, et al. Complications associated with liver transplantation in the obese recipient. Clin Transplant 2012;26:910-8.

10. Hotouras A, Ribas Y, Zakeri SA, et al. The influence of obesity and body mass index on the outcome of laparoscopic colorectal surgery: a systematic literature review. Colorectal Dis 2016;18:0337-066.
11. Muelleman T, Shew M, Muelleman RJ, et al. Obesity Does Not Increase Operative Time in Otologic Surgery: An Analysis of 5125 Cases. Otol Neurotol 2018;39:e103-e7.

12. Hillings $\varnothing J G$, Wettergren A, Hyoudo M, Kirkegaard P. Obesity increases mortality in liver transplantation-the Danish experience. Transpl Int 2005;18:1231-5.

13. Sawyer RG, Pelletier SJ, Pruett TL. Increased early morbidity and mortality with acceptable long-term function in severely obese patients undergoing liver transplantation. Clin Transplant 1999;13:126-30.

14. Nair S, Cohen DB, Cohen MP, Tan H, Maley W, Thuluvath PJ. Postoperative morbidity, mortality, costs, and long-term survival in severely obese patients undergoing orthotopic liver transplantation. Am J Gastroenterol 2001;96:842-5.

15. Braunfeld MY, Chan S, Pregler J, et al. Liver transplantation in the morbidly obese. J Clin Anesth 1996;8:585-90.

16. Feltracco P, Brezzi M, Barbieri S, et al. Blood loss, predictors of bleeding, transfusion practice and strategies of blood cell salvaging during liver transplantation. World J Hepatol 2013;5:1-15.

17. Tripodi A, Mannucci PM. The coagulopathy of chronic liver disease. N Engl J Med 2011;365:147-56.

18. Massicotte L, Sassine MP, Lenis S, Roy A. Transfusion predictors in liver transplant. Anesth Analg 2004;98:1245-51.

19. Hannaman MJ, Hevesi ZG. Anesthesia care for liver transplantation. Transplant Rev (Orlando) 2011;25:36-43.

20. Tovikkai C, Charman SC, Praseedom RK, Gimson AE, van der Meulen J. Time spent in hospital after liver transplantation: Effects of primary liver disease and comorbidity. World J Transplant 2016;6:743-50.

21. Amiri M, Toosi MN, Moazzami B, et al. Factors Associated With Length of Hospital Stay Following Liver Transplant Surgery. Exp Clin Transplant 2020;18:313-9.

22. Leonard J, Heimbach JK, Malinchoc M, Watt K, Charlton $M$. The impact of obesity on long-term outcomes in liver transplant recipients-results of the NIDDK liver transplant database. Am J Transplant 2008;8:667-72. 